\title{
ON THE DEGREE OF APPROXIMATION OF FUNCTIONS BELONGING TO THE LIPSCHITZ CLASS BY $(e, c)$ MEANS
}

\author{
U. K. SHRIVASTAVA AND S. K. VERMA
}

\begin{abstract}
In the present paper, we obtain the degree of approximation of $f \varepsilon$ Lip $\alpha$ $(0<\alpha \leq 1)$ by $(e, c)$ means $(c>0)$ of its Fourier Series.
\end{abstract}

\section{Introduction}

Let $C_{2 \pi}$ be the space of all $2 \pi$-periodic and continuous functions defined on $[-\pi,+\pi]$, which is a Banach space under the "sup" norm.

A function $f \varepsilon \operatorname{Lip} \alpha(0<\alpha \leq 1)$ if

$$
f(x+h)-f(x)=O\left(|h|^{\alpha}\right)
$$

For each $f \varepsilon c_{2 \pi}$ let the Fourier series be given by

$$
S(x)=\sum_{m=-\infty}^{\infty} C_{m} \exp (i m x)
$$

where, $C_{m^{\prime} s}$ are Fourier coefficients. Let the $n^{\text {th }}$ partial sum of the series (2) be

$$
S_{n}(f ; x)=\sum_{m=-n}^{n} C_{m} \exp (i m x)
$$

A series $\sum_{n=0}^{\infty} a_{n}$ with the sequence of partial sum $\left\{S_{n}\right\}$ is said to be summable $(e, c)$, $(c>0)$ if $($ see $[4])$

$$
\lim _{n \rightarrow \infty} t_{n}^{c}=\lim _{n \rightarrow \infty} \sqrt{\frac{c}{\pi n}} \sum_{k=-\infty}^{\infty} \exp \left(-\frac{c k^{2}}{n}\right) S_{n+k}
$$

Received January 18, 1994. 
exists, where it is to be understood that $S_{n+k}=0$, when $n+k<0$.

The $(e, c)$ summability method is regular for $c>0$. It is interesting to note that this method includes several other methods of summability, namely, Borel, $(E, q),(\gamma, k)$ etc. (For details see Hardy [3] Theorem 159).

We shall write

$$
\left\|t_{n}^{c}-f\right\|=\sup _{-\pi \leq x \leq \pi}\left|t_{n}^{c}(f ; x)-f(x)\right|
$$

where $t_{n}^{c}(f ; x)$ is $n^{\text {th }}(e, c)$-means of the Fourier series of $f$ at $x$.

Degree of approximation by Borel means and $(E, q)$-means were obtained by Chandral [1]\&[2] respectively. Since $(e, c)$-method includes $(B, \alpha)$ and $(E, q)$ method, it is natural to ask, what will be the result if we apply $(e, c)$-means to obtain the degree of approximation for $f \in \operatorname{Lip} \alpha(0<\alpha \leq 1)$ ?

We shall prove the following theorem.

Theorem: Let $f \varepsilon c_{2 \pi} \cap \operatorname{Lip} \alpha, 0<\alpha \leq 1$ Then

$$
\left\|t_{n}^{c}-f\right\|=O\left(n^{-\alpha / 2}\right)
$$

\section{Inequalities}

In the proof of our theorem, we shall use the following inequalities

$$
\begin{aligned}
& \sum_{k=n+1}^{\infty} k \exp \left(-\frac{c k^{2}}{n}\right) \leq \frac{n}{2 c} \exp (-c n) \\
& \left|\sum_{k=n+1}^{\infty} \exp \left(-\frac{c k^{2}}{n}\right) \sin \left(n+k+\frac{1}{2}\right) t\right| \leq \frac{n t}{2 c} \exp (-c n) \\
& \sum_{k=n+1}^{\infty} \exp \left(-\frac{c k^{2}}{n}\right) \cos (k t)=O\left\{\frac{\exp (-c n)}{t}\right\} \\
& 1+2 \sum_{k=1}^{\infty} \exp \left(-\frac{c k^{2}}{n}\right) \cos (k t)=\sqrt{\frac{\pi n}{c}}\left\{\exp \left(-\frac{n t^{2}}{4 c}\right)+O\left(\exp \left(-\frac{n \pi}{4 c}\right)\right)\right\}
\end{aligned}
$$

The inequality (6) follows from (5), (7) may be obtained by using Abel's Lemma and (8) may be obtained by the classical formula for theta function (see Siddiqui [5]). Thus we prove only (5).

Proof of (5) We observe that $Y \exp \left(-\frac{c \gamma^{2}}{n}\right)$ is non-increasing for $Y \geq \sqrt{\frac{n}{2 c}}$ and 
hence

$$
\begin{aligned}
\sum_{k=n+1}^{\infty} k \exp \left(-\frac{c k^{2}}{n}\right) & \leq \int_{n}^{\infty} Y \exp \left(\frac{c \gamma^{2}}{n}\right) d \gamma \\
& =\frac{n}{2 c} \int_{n}^{\infty} \frac{d}{d \gamma}\left(-\exp \left(-\frac{c \gamma^{2}}{n}\right)\right) d \gamma \\
& =\frac{n}{2 c} \exp (-c n)
\end{aligned}
$$

This completes the proof of (5)

\section{Proof of Theorem}

Following Titchmarsh [6] p.403, we have

$$
S_{n}(f ; x)-f(x)=\frac{1}{\pi} \int_{0}^{\pi} \frac{\varphi_{x}(t)}{\sin \left(\frac{t}{2}\right)} \sin \left(n+\frac{1}{2}\right) t d t
$$

where

$$
2 \varphi_{x}(t)=f(x+t)+f(x-t)-2 f(x)
$$

then

$$
\begin{aligned}
& t_{n}^{c}(f ; x)-f(x)=\frac{1}{\pi} \sqrt{\frac{c}{\pi n}} \int_{0}^{\pi} \frac{\varphi_{x}(t)}{\sin \left(\frac{t}{2}\right)}\left[\sum_{k=-n}^{\infty} \exp \left(-\frac{c k^{2}}{n}\right) \sin \left(n+k+\frac{1}{2}\right) t\right] d t \\
= & \frac{1}{\pi} \sqrt{\frac{c}{\pi n}} \int_{0}^{\pi} \frac{\varphi_{x}(t)}{\sin \left(\frac{t}{2}\right)}\left[\left\{1+2 \sum_{k=1}^{n} \exp \left(-\frac{c k^{2}}{n}\right) \cos (k t)\right\} \sin \left(n+\frac{1}{2}\right) t\right. \\
& \left.+\sum_{k=n+1}^{\infty} \exp \left(-\frac{c k^{2}}{n}\right) \sin \left(n+k+\frac{1}{2}\right) t\right] d t \\
= & \frac{1}{\pi} \sqrt{\frac{c}{\pi n}}\left[\int _ { 0 } ^ { \pi } \frac { \varphi _ { x } ( t ) } { \operatorname { s i n } ( \frac { t } { 2 } ) } \left[\left\{1+2 \sum_{k=1}^{n} \exp \left(-\frac{c k^{2}}{n}\right) \cos (k t)\right\} \sin \left(n+\frac{1}{2}\right) t .\right.\right. \\
& \left.\left.-2 \sum_{k=n+1}^{\infty} \exp \left(-\frac{c k^{2}}{n}\right) \cos (k t) \sin \left(n+\frac{1}{2}\right) t+\sum_{k=n+1}^{\infty} \exp \left(-\frac{c k^{2}}{n}\right) \sin \left(n+k+\frac{1}{2}\right) t\right] d t\right] \\
= & I_{1}+I_{2}+I_{3}, \operatorname{say}
\end{aligned}
$$

Hence

$$
\left\|t_{n}^{c}(f)-f\right\| \leq\left\|I_{1}\right\|+\left\|I_{2}\right\|+\left\|I_{3}\right\|
$$

Now

$$
\left\|I_{3}\right\|=O\left(n^{-1 / 2}\right) \int_{0}^{\pi} t^{\alpha-1}\left|\sum_{k=n+1}^{\infty} \exp \left(-\frac{c k^{2}}{n}\right) \sin \left(n+k+\frac{1}{2}\right) t\right| d t
$$




$$
\begin{aligned}
& =O\left(n^{1 / 2}\right) \int_{0}^{\frac{\pi}{n}} t^{\alpha} \exp (-c n) d t+O\left(n^{-1 / 2}\right) \int_{\frac{\pi}{n}}^{\pi} t^{\alpha-2} \exp (-c n) d t \\
& =O\left(n^{-\frac{1}{2}-\alpha} \exp (-c n)\right)+O\left(n^{-\frac{1}{2}}\right) \exp (-c n) \begin{cases}n^{1-\alpha} & \text { for } 0<\alpha<1 \\
\log n & \text { for } \alpha=1\end{cases} \\
& = \begin{cases}O\left(n^{\frac{1}{2}-\alpha} \exp (-c n)\right) & \text { for } 0<\alpha<1 \\
O\left(n^{-\frac{1}{2}} \exp (-c n) \log n\right) & \text { for } \alpha=1\end{cases}
\end{aligned}
$$

Similarly

$$
\left\|I_{2}\right\|= \begin{cases}O\left(n^{\frac{1}{2}-\alpha} \exp (-c n)\right) & \text { for } 0<\alpha<1 \\ O\left(n^{-\frac{1}{2}} \exp (-c n) \log n\right) & \text { for } \alpha=1\end{cases}
$$

Finally by (8), we have

$$
\begin{aligned}
I_{1} & =\int_{0}^{\pi} \frac{\varphi_{x}(t)}{\sin (t / 2)} \sin \left(n+\frac{1}{2}\right) t \exp \left(-\frac{n t^{2}}{4 c}\right) d t+O(1) \int_{0}^{\pi} \frac{\mid \varphi_{x}(t)}{\sin (t / 2)}\left|\sin \left(n+\frac{1}{2}\right) t\right| \exp \left(-\frac{n \pi}{4 c}\right) d t \\
& =I_{1,1}+I_{1,2} \text { say }
\end{aligned}
$$

Now

$$
\begin{aligned}
\left\|I_{1,2}\right\| & =O\left(\exp \left(-\frac{n \pi}{4 c}\right)\right)\left[\int_{0}^{\frac{\pi}{n}}+\int_{\frac{\pi}{n}}^{\pi}\right] t^{\alpha-1}\left|\sin \left(n+\frac{1}{2}\right) t\right| d t \\
& =O\left\{n^{-\alpha} \exp \left(-\frac{n \pi}{4 c}\right)\right\}+O\left\{\exp \left(-\frac{n \pi}{4 c}\right)\right\}
\end{aligned}
$$

and

$$
\begin{aligned}
\left\|I_{1,1}\right\| & =O(1) \int_{0}^{\pi} t^{\alpha-1} \exp \left(-\frac{n t^{2}}{4 c}\right)\left|\sin \left(n+\frac{1}{2}\right) t\right| d t \\
& =O(1) \int_{0}^{\frac{\pi}{\sqrt{n}}} t^{\alpha-1} d t+O\left(n^{-1}\right) \int_{\frac{\pi}{\sqrt{n}}}^{\pi} t^{\alpha-2} \frac{\partial}{\partial t}\left[\exp \left(-\frac{n t^{2}}{4 c}\right)\right] d t \\
& =O\left(n^{-\alpha / 2}\right)
\end{aligned}
$$

Collection of (9), (10), .. (14) completes the proof of the theorem.

\section{Acknowledgment}

We are thankful to Dr. Prem Chandra, Professor, School of Mathematics, Vikram University Ujjain and Dr. R. S. yadav, Head, Department of Mathematics, Govt. Model Science College Bilaspur for their valuable guidance during the preparation of this paper. 


\section{References}

[1] Prem Chandra.. "On the Degree of Approximations of Functions Belonging to the Lipschitz class," La.b Dev. J. Science and Technology, 13A (1975), 181-183.

[2] Prem Chandra. "On the Degree of Approximation of Continuous functions," Commun. Fac. Sci. Univ. Ankara, Ser A, 30(1981), 7-16.

[3] G. H. Hardy, Divergent Series, Oxford University Press (1949).

[4] G. H. Hardy and J. E. Littlewood, "Theorems concerning the summability of series by Borel's exponential method," Rend. Circ. Mat. Palermo 41 (1916), 36-53.

[5] Jamil A. Siddiqui, "A criterion for the $(e, c)$ summability of Fourier series," Proc. Camb. Phil Soc. 92 (1982), 121-127.

[6] E. C. Titchmarsh, A theory of Function, Oxford University Press (1978).

Department of Mathematics, Govt. Girls P. G. College, Bilaspur (M. P.), India 495001. 